Informes de la Construcción Vol. 62, 519, 55-66 julio-septiembre 2010 ISSN: 0020-0883 eISSN: 1988-3234 doi: 10.3989/ic.09.036

\title{
Caracterización térmica de placas de yeso con material de cambio de fase incorporado
}

\section{Thermal characterization of gypsum boards with PCM}

\author{
A. Oliver ${ }^{(*)}$, F. J. Neila ${ }^{(*)}$, A. García ${ }^{(*)}$
}

\section{RESUMEN}

En este trabajo se demuestra la idoneidad de incorporar materiales de cambio de fase en placas de yeso para incrementar su capacidad de almacenamiento térmico. Para ello se evalúa y se compara la capacidad de almacenamiento térmico, de diferentes elementos constructivos cuyo uso y aplicación es similar a la de las placas de yeso: trasdosado y tabique separador.

Se ha diseñado y puesto en funcionamiento una instalación experimental que simula las condiciones de contorno que se producen en una estancia donde estén instalados los diferentes materiales y sistemas constructivos. Se ha estudiado la influencia de diferentes parámetros y variables del sistema (temperatura de trabajo, velocidad del aire, presentación de los materiales de cambio de fase, ubicación en el edificio,...), para constituir un sistema de almacenamiento de calor latente, que, complementado con estrategias pasivas (captación solar, ventilación natural), reduzca las necesidades de consumo energético para la climatización de edificios.

Se obtiene que las placas de yeso con un $45 \%$ en peso de material de cambio de fase es capaz de almacenar en $1,5 \mathrm{~cm}$ de espesor, 5 veces la energía térmica de un panel de yeso laminado con el mismo espesor, y la misma cantidad que $1 / 2$ pie de fábrica ladrillo hueco sencillo, en el rango de temperaturas próximas a la de confort $\left(20-30{ }^{\circ} \mathrm{C}\right)$, manteniendo las propiedades físicas y mecánicas exigidas en la normativa

$614-19$

Palabras clave: material de cambio de fase, yeso, placa, energía térmica, almacenamiento.

\section{SUMMARY}

In this research the suitability of including PCM in gypsum boards to multiply their thermal energy storage capacity is demonstrated. So the thermal storage capacity of several constructive materials is evaluated and compared, whose use and application is similar to the gypsum boards: paneling of a wall and partition wall.

An experimental facility has been designed and operated for the study of the new compound material, exchanging heat with air. It has been studied the influence of different parameters and system variables (working temperature, air velocity, display of the phase change materials, and location in the building...), to establish a latent heat storage system which-complemented with passive strategies (solar gains, natural ventilation)reduces the acclimatization energy consumption in buildings.

So, here we demonstrate that a gypsum board including a $44.5 \%$ in weight of phase change materials (PCM) is able to store -in a $1.5 \mathrm{~cm}$ thick5 times the thermal energy of a current gypsum board -with the same thickness-, and the same amount to $11.5 \mathrm{~cm}$ brick layer, in the comfort temperature range $\left(20-30{ }^{\circ} \mathrm{C}\right)$, maintaining the mechanical and physical properties required by the regulations in force.

Keywords: PCM, gypsum, board, thermal energy, storage.

\footnotetext{
(*) Dpto. Construcción y Tecnología Arquitectónicas. ETS de Arquitectura (E.T.S.), Madrid (España) Persona de contacto/Corresponding author: oliver_alice@yahoo.es (A. Oliver)
} 


\author{
ABREVIATURAS \\ PF: Placas finas $(1,5 \mathrm{~cm}=\mathrm{e})$ \\ PG: Placas gruesas $(2,5 \mathrm{~cm}=\mathrm{e})$ \\ CY: Placa de yeso laminado \\ LH: Fábrica de ladrillo hueco $(\mathrm{e}=11,5 \mathrm{~cm})$ \\ PCM: Phase Change Material, material de \\ cambio de fase \\ T: Posición trasdosada \\ E.O.: Posición exenta-oblicua
}

\section{ANTECEDENTES}

La necesidad de buscar formas de acondicionamiento pasivo, en que el ahorro energético tenga una componente importante es fundamental, ya que no sólo permite reducir gastos, sino también limitar el uso de los combustibles tradicionales (1).

En los últimos años los sistemas de almacenamiento de energía térmica están suscitando un gran interés ya que nos permite adaptar los periodos de suministro a los de demanda energética, por lo que constituyen un gran potencial para la mejora de la eficiencia energética. Entre sus múltiples aplicaciones relacionadas con el ahorro energético están:

- Reserva de los excedentes térmicos y energías renovables (solar, eólica,...) de forma gratuita -tanto para calefacción como refrigeración- y utilizarlos en el momento preciso, diferente al de suministro.

- Reducción y moderación de la demanda eléctrica evitando los consumos pico.

Los Materiales de Cambio de Fase son una de las formas más eficientes de almacenamiento térmico. Se conocen internacionalmente por su acrónimo en inglés PCM -Phase Change Material-.

El interés de este tipo de materiales está en que, durante el cambio de fase, la temperatura se mantiene constante mientras que el material absorbe energía. Este hecho supone una mayor densidad energética de estos materiales en comparación con cualquier otro. Otro aspecto muy positivo es la menor temperatura a la cual se acumula la energía, consiguiendo, por lo tanto, unas pérdidas menores (8).

Los materiales de cambio de fase almacenan energía en forma de calor latente y poseen una elevada densidad de almacenamiento térmico, independiente de la variación de temperatura (16) (18). Son muchas sus aplicaciones en diversos campos (sanitario, botánico,...) (4) (9). En el campo de la construcción desde inicios de los años 80 se están realizando diversos trabajos de investigación para incorporarlos a diferentes elementos constructivos (hormigón, yeso, cerámica, vidrio,...) con el fin de mejorar su inercia térmica (2) (7) (10) (11).

En España se ha realizado una investigación basada en la incorporación de estos materiales en módulos prefabricados de hormigón (17), y se está investigando en la integración de PCM en ladrillo perforado.

Se ha elegido el yeso como material más adecuado para desarrollar un nuevo producto de construcción por su disponibilidad, profusa utilización en el campo de la edificación y bajo coste.

Existen numerosas investigaciones que se centran en la incorporación PCM en placas de yeso. Unas se centran en la caracterización física a nivel constructivo como los trabajos aportados en (6) y (14), y otras en el cálculo numérico de la capacidad térmica como en (5) (12) (15) y (19). Comercialmente existe un producto basado en la incorporación de PCM a paneles de yeso laminado -Smart Board, de BASF- (3) que incorpora un $26 \%$ en peso del material, es decir, $3 \mathrm{~kg} /$ m2 de panel, con 1,5 cm de espesor. Esta cantidad es la mayor incorporada hasta el presente trabajo, a una placa de yeso.

El campo de interés de este trabajo, en cuanto a la acumulación térmica, se centra en la aplicación en los materiales de construcción -yesos-, para reducir incluso eliminar -según las condiciones climáticas a que esté sometida- las cargas de calefacción y refrigeración.

\section{ENSAYOS}

Se propone un método sencillo para evaluar el comportamiento térmico de los diferentes materiales constructivos: la capacidad de almacenamiento térmico, el tiempo de respuesta, efectividad del sistema, según las condiciones de contorno (velocidad del aire, temperatura de la sala...).

Como material de cambio de fase se ha elegido una parafina microencapsulada -que evita el problema de fugas durante la fase líquida y favorece la transferencia de calor por el incremento de la superficie de contacto-. De todas las parafinas disponibles -a nivel comercial, y a nivel de laboratorio- se ha elegido Micronal DS 5001X, fabricada y comercializada por BASF, cuya temperatura de cambio de fase es de $26{ }^{\circ} \mathrm{C}$, y la entalpía asociada es de unos $110 \mathrm{~J} / \mathrm{g}$ (Figura 1).

Esta parafina se ha mezclado con yeso y con otros aditivos de refuerzo -fibras y dispersión- para conformar placas de este material 


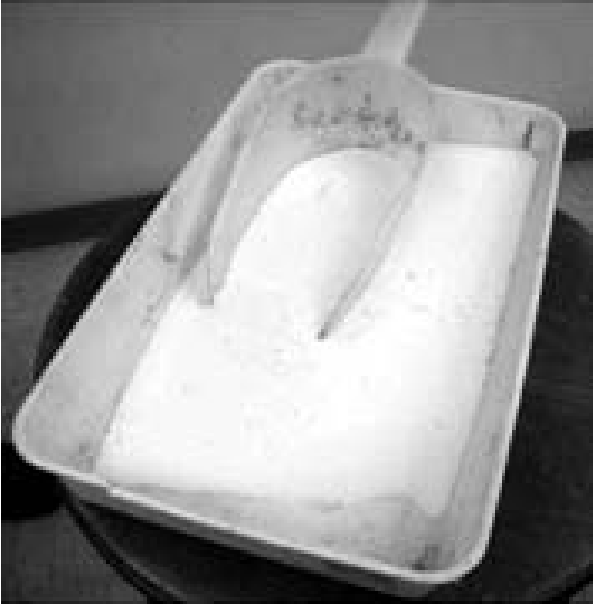

compuesto. Se han utilizado diferentes dosificaciones de cada agregado para obtener diferentes compuestos los cuales se han ensayado física y mecánicamente.

De todos los compuestos fabricados se ha elegido el compuesto que contiene el mayor porcentaje de material de cambio de fase incorporado, y cumple con la normativa de aplicación respecto a la caracterización física y mecánica de un producto derivado del yeso (UNE 13279) (13).

Este compuesto está formado por la siguiente dosificación:

- $100 \%$ de yeso (Escayola E-30)

- $100 \%$ de agua

- $80 \%$ de material de cambio de fase, Micronal DS 5001X

- $3,5 \%$ de fibra de polipropileno

- 4,0\% de dispersión, melamina formaldehído

Además de las placas fabricadas por este compuesto con dos espesores $(1,5$ y $2,5 \mathrm{~cm})$, se han estudiado diversos materiales y sistemas constructivos, utilizados en construcción que desempeñan la misma función que la propuesta para las placas de yeso con material de cambio de fase, es decir, como hoja interior de la fachada, o como sistema de tabiquería interior.

Los materiales constructivos ensayados son:

- Placa de yeso con PCM (44,5\%) (e = $1,5 \mathrm{~cm})$

- Placa de yeso con PCM (44,5\%) (e = $2,5 \mathrm{~cm})$

- Placa de Yeso laminado $(\mathrm{e}=1,5 \mathrm{~cm})$

- Fábrica de ladrillo hueco ( $\mathrm{e}=11,5 \mathrm{~cm}$ )

- Fábrica de termoarcilla $(\mathrm{e}=14,0 \mathrm{~cm})$.

El estudio se ha hecho en ambas posiciones (trasdosada y exenta), y a dos temperaturas

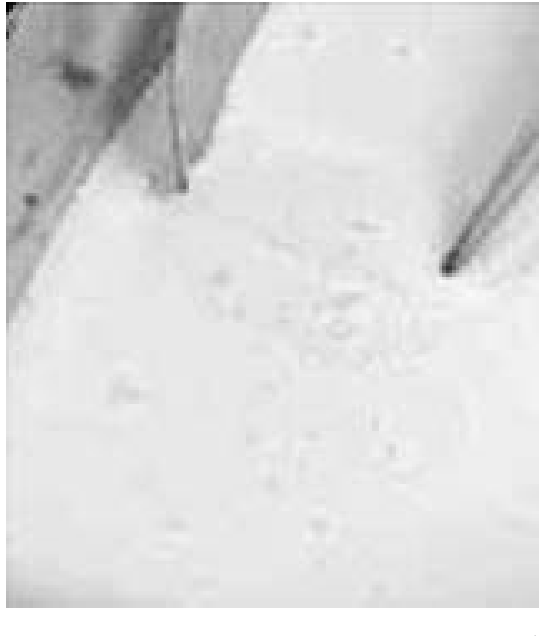

Muestra del material de cambio de ase-Micronal DS 5001X_utilizado en el laboratorio para la fabricación dev placas de yeso con PCM diferentes de entrada de aire (Te) 30 y $35^{\circ} \mathrm{C}$. En la Figura 4 se muestra una imagen de las placas de yeso con PCM colocadas en posición trasdosada dentro del depósito intercambiador del equipo experimental.

Se realizan gráficos de temperatura-tiempo, energía almacenada-tiempo, y se evalúan las propiedades por comparación entre los distintos materiales sometidos a los mismos ensayos.

Se ha diseñado y construido una instalación experimental para la realización de estos ensayos que simula en el laboratorio -de manera controlada- las condiciones de intercambio de energía entre los materiales y el ambiente interior.

La instalación consiste básicamente en un circuito cerrado de aire, movido por un pequeño sistema de impulsión mediante un ventilador regulable, que en una etapa es calentado hasta las condiciones seleccionadas para el ensayo y en otra etapa se realiza el experimento propiamente dicho, intercambiando calor con los materiales de ensayo. Se disponen medidores de temperatura y caudal, los cuales permiten medir la diferencia entre la temperatura de entrada y de salida del aire en el intercambiador, con la que podemos obtener la energía acumulada en cada intervalo de tiempo, $y$, a partir de esta magnitud, integrando, la energía que se ha almacenado o cedido desde el comienzo del proceso de intercambio de calor con el aire.

Los principales componentes de la instalación experimental son: Caja adiabática, Conductos para el circuito cerrado de aire, ventilador, regulador de velocidad para el ventilador, resistencia, regulador PID para la resistencia, caudalímetro y estabilizador de tensión, Sensores de temperatura, Sistema de toma de datos, Programa de toma de da- 
2. Esquema del equipo experimental (elaboración propia).

3. Imagen del equipo experimental utilizado en las mediciones térmicas.

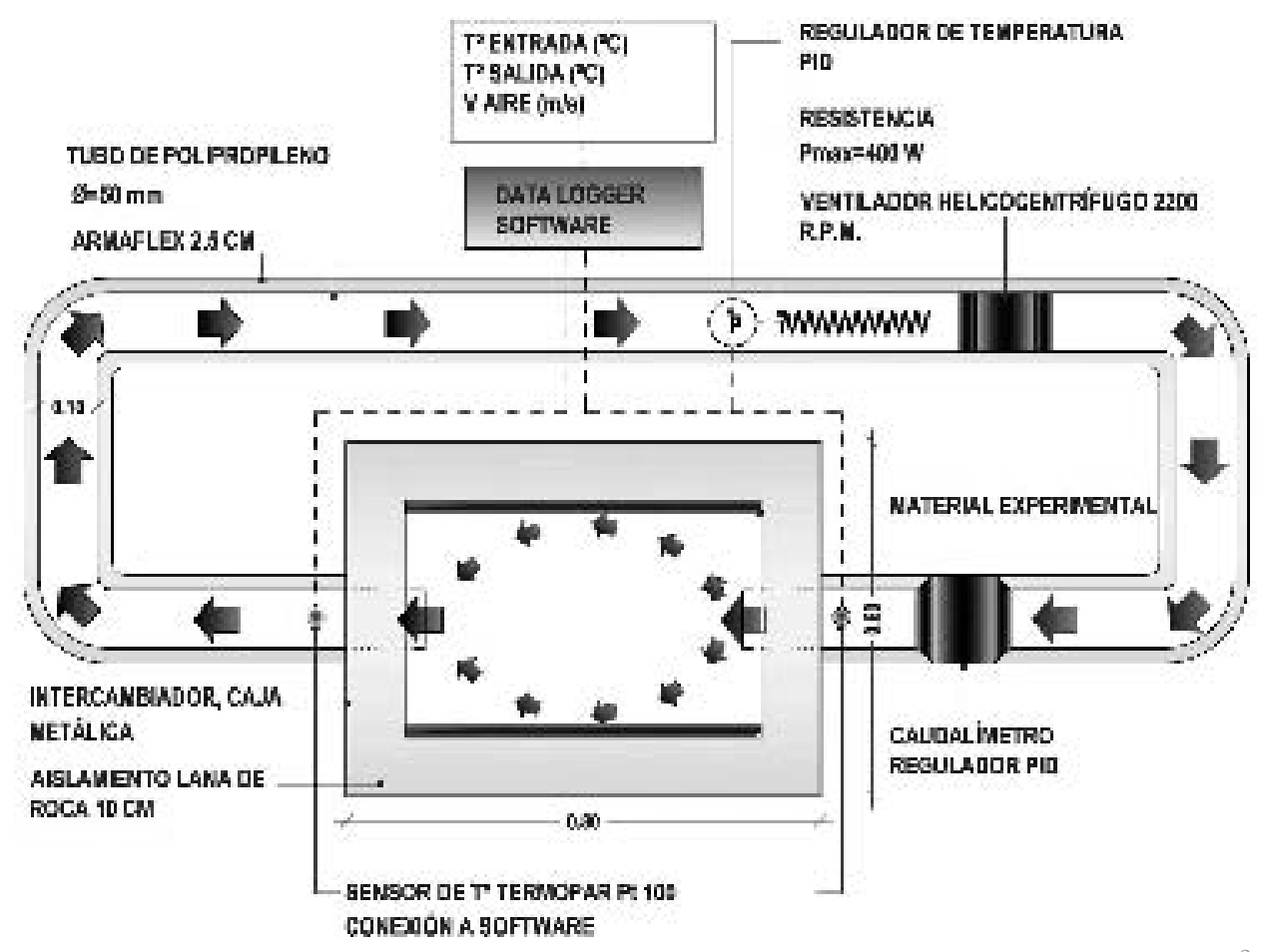

espesor propio $(1,5 \mathrm{~cm}$ y $2,5 \mathrm{~cm}$ para los dos tipos de placa, $1,5 \mathrm{~cm}$ para el yeso laminado, $11,5 \mathrm{~cm}$ para el ladrillo hueco, y $14 \mathrm{~cm}$ para la termoarcilla. Hay que observar que la cantidad de masa $\left(\mathrm{kg} / \mathrm{m}^{2}\right)$ de cada uno de ellos es diferente.

Esta comparación se va a hacer a dos temperaturas diferentes $\left(30\right.$ y $\left.35^{\circ} \mathrm{C}\right)$, y para dos posiciones diferentes (trasdosada y exenta). La primera intercambia energía sólo por una de sus caras, y, para la segunda, la transferencia es por ambas.

Para un sistema trasdosado, y temperatura de trabajo de $30{ }^{\circ} \mathrm{C}$, se han tomado valores de temperatura de entrad y de salida de aire. En la Gráfica 1 se muestran los resultados obtenidos en la variación de Te, Ts, Tint, y Tsup, para uno de los ensayos realizados.

A partir de los valores obtenidos para se puede obtener la evolución de la diferencia entre la temperatura de entrada y de salida con la ecuación [1]. Los resultados obtenidos se representan en la Gráfica 2.

$\Delta(T e-T S)=\frac{\left(T e_{n}-T S_{n}\right)+\left(T e_{n-1}-T S_{n-1}\right)}{2}$

tos y Material de ensayo (13) Su esquema de funcionamiento se representa en la Figura 2. En la Figura 3 se muestra una imagen de la instalación experimental.

\section{ANÁLISIS DE RESULTADOS DE LOS ENSAYOS}

\subsection{Comparación entre distintos materiales}

Se va a considerar $1 \mathrm{~m}^{2}$ de superficie de cada uno de los materiales, cada uno con su
Operando con estos valores y utilizando la expresión [2], se obtiene la energía acumulada por intervalos, cuyos valores se representan en la Gráfica 3.

$$
\mathrm{Q}_{\text {almacenado n }}=\mathrm{Q}_{\text {almacenado n-1 }}+
$$


La energía almacenada en el tiempo se obtiene integrando la expresión [2] puesto que se corresponde con el área de cada gráfica (la comprendida entre el trazo de la gráfica y el eje X) Los valores para cada uno de los materiales se muestran en la Gráfica 4, pág. siguiente.

La Tabla 1 (pág. siguiente) recoge los valores obtenidos en la Gráfica 4, a las 2 y 4 horas de inicio del ensayo.

Del análisis de las gráficas obtenidas para cada uno de los materiales ensayado se ob-

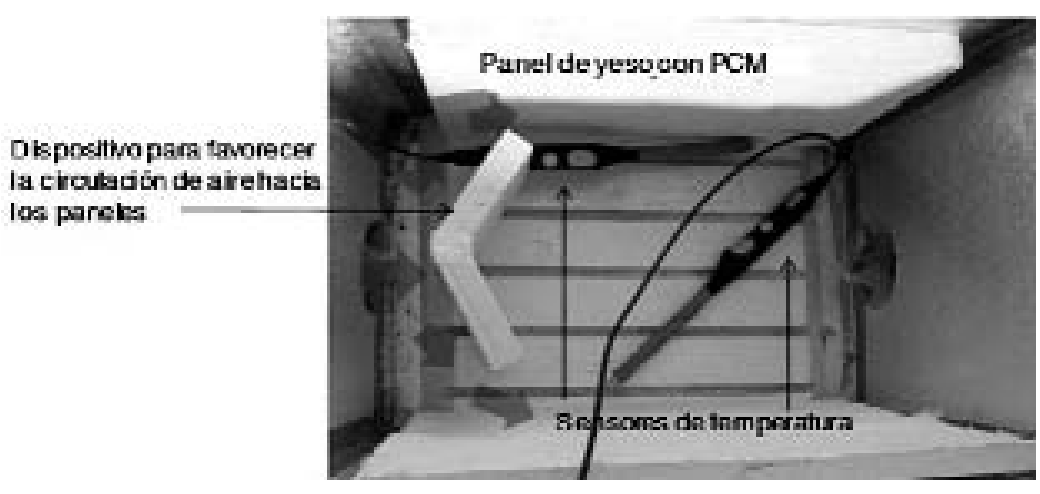

Gráfica 1. Evolución de Ts, Tsup y Tint para $\mathrm{Te}=35^{\circ} \mathrm{C}$, con placas.

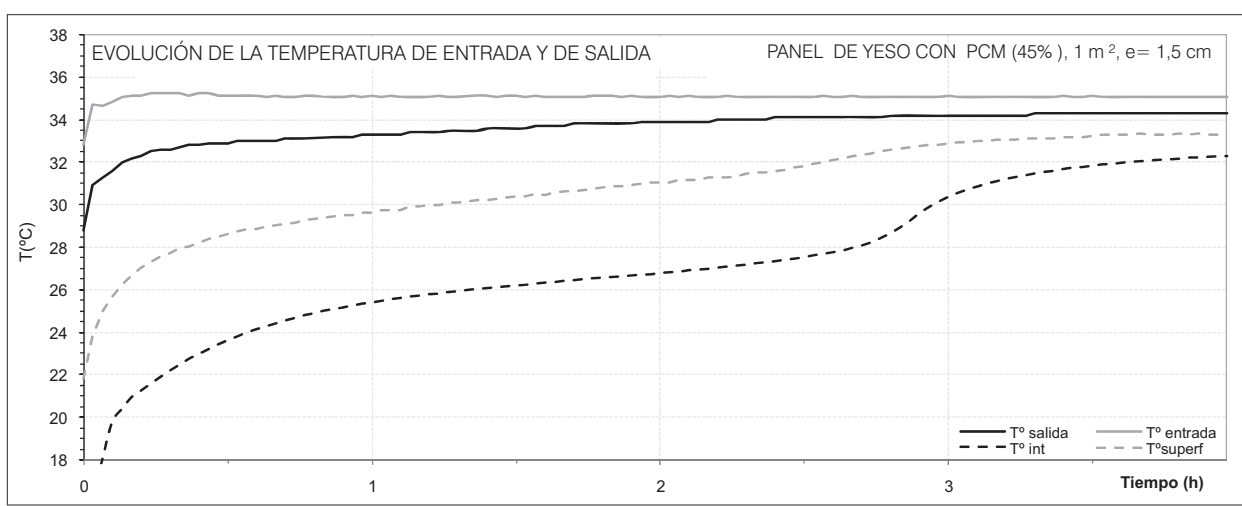

4. Imagen del material de ensayo -placas de yeso con PCM- colocado en el interior de la caja intercambiadora del equipo para los ensayos térmicos.

Gráfica 2. Comparación de la evolución de la diferencia entre Te y Ts para $1 \mathrm{~m}^{2}$ de superficie de diferentes materiales constructivos. (PF, placas finas, $\mathrm{e}=\mathbf{1 , 5} \mathrm{cm} ; \mathrm{PG}$, placas gruesas, $\mathrm{e}=2,5 \mathrm{~cm}$; LHD, ladrillo hueco doble, $\mathrm{e}=1,5 \mathrm{~cm}$; $\mathrm{C}$ Yeso, Yeso laminado, $\mathrm{e}=1,5 \mathrm{~cm})\left(\mathrm{Te}, 30^{\circ} \mathrm{C}\right)$. Posición adosada.

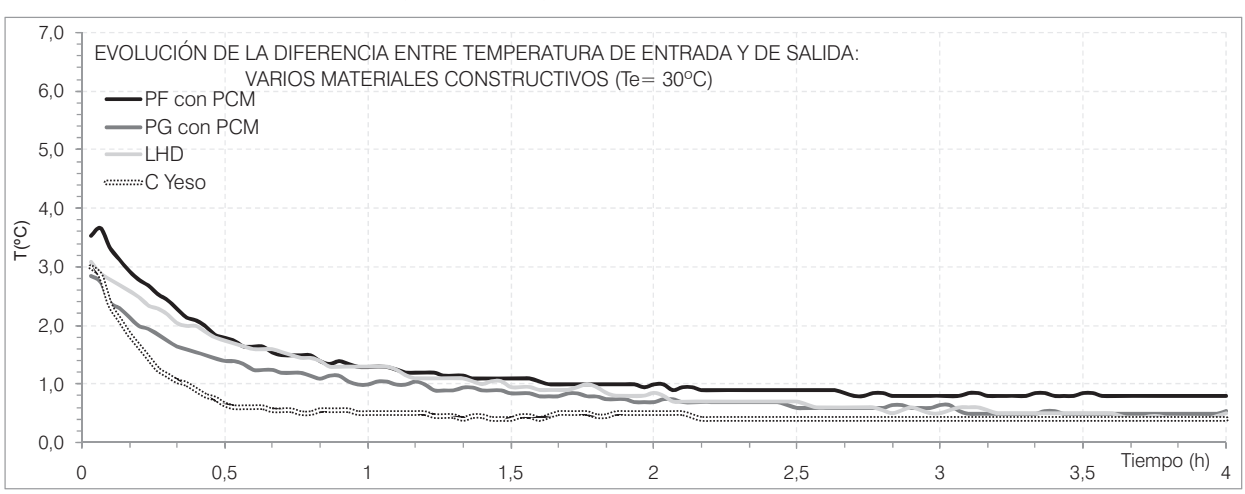

Gráfica 3. Comparación de energía acumulada por intervalos, para varios materiales constructivos $\left(\mathrm{Te}, 30^{\circ} \mathrm{C}\right)$. Posición adosada

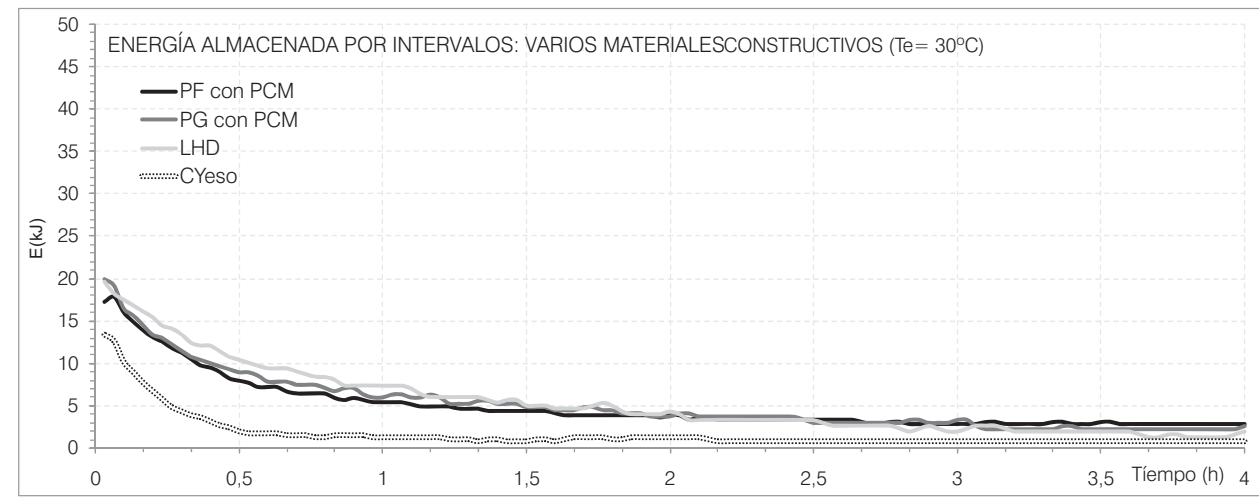


Gráfica 4. Comparación de energía acumulada por intervalos, para varios materiales constructivos $\left(\mathrm{Te}, 30^{\circ} \mathrm{C}\right.$ ). Posición adosada

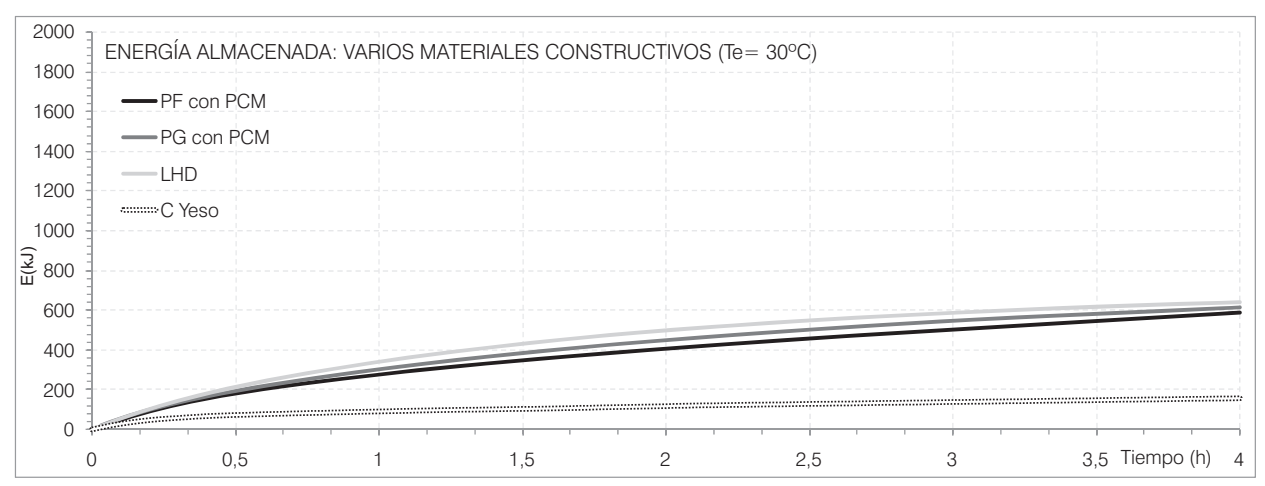

Tabla 1

Comparación de energía acumulada por intervalos, para varios materiales constructivos (Te, $30^{\circ} \mathrm{C}$ ). Posición adosada

\begin{tabular}{|c|c|c|}
\hline \multicolumn{3}{|c|}{ Energía almacenada (kJ) } \\
\hline \multirow{2}{*}{ Material } & \multicolumn{2}{|c|}{ Tiempo } \\
\hline & $2 \mathrm{~h}$ & $4 \mathrm{~h}$ \\
\hline PF & 407,54 & 587,55 \\
\hline PG & 450,86 & 617,05 \\
\hline CY & 119,63 & 160,20 \\
\hline LH & 500,53 & 641,69 \\
\hline
\end{tabular}

serva que las funciones de PF, PG, y LHD, discurren muy próximas durante todo el periodo de ensayo, en las tres gráficas. Ello significa que el comportamiento térmico es muy similar. La función de CY, es claramente diferente a las anteriores. Su capacidad de almacenamiento térmico es muy inferior a las anteriores, por lo que para un mismo aporte de energía del sistema, colapsa rápidamente (apenas 20 minutos), permitiendo que la temperatura de salida Ts, se eleve a su valor máximo en ese momento.
A $30^{\circ}$ de temperatura de trabajo, la fábrica de ladrillo hueco almacena un 4,0\% más de energía que las placas de $2,5 \mathrm{~cm}$ de espesor. Las placas de 1,5 cm almacenan un 4,8\% menos y el yeso laminado apenas almacena un $32,5 \%$ respecto a las placas de yeso con PCM de 2,5 cm de espesor)

A esta misma temperatura, en posición exenta oblicua los resultados obtenidos se representan en las Gráficas 5, 6, 7 y 8.

En la Tabla 2 se reproducen los valores obtenidos en la Gráfica 8, a las 2 y 4 horas de inicio del ensayo.

En posición exenta, para la que se recibe energía por ambas caras del sistema constructivo, una placa de yeso con PCM de 2,5 cm de espesor posee una capacidad de almacenamiento térmico, un $15,9 \%$ inferior a la de una hoja de fábrica de termoarcilla (T), y un $12,1 \%$ más que la misma placa de $1,5 \mathrm{~cm}$ de espesor, tal y como se describe en la Tabla 3. Hay que recordar que para construir $1 \mathrm{~m}^{2}$ de fábrica de termoarcilla se necesitan $140 \mathrm{~kg}$, muy superiores a los $17,8 \mathrm{~kg}$ que son necesarios para configurar una placa de $2,5 \mathrm{~cm}$ de espesor de yeso con mate-

Gráfica 5. Evolución de Ts, para $1 \mathrm{~m}^{2}$ de superficie de varios materiales constructivos (PF, placas finas, $\mathrm{e}=1,5, \mathrm{PG}$, placas gruesas, $\mathrm{e}=2,5 \mathrm{~cm}$, ambos de escayola con Micronal al 44,5\%; Termoarcilla) (Te, $\left.30^{\circ} \mathrm{C}\right)$. Posición exenta

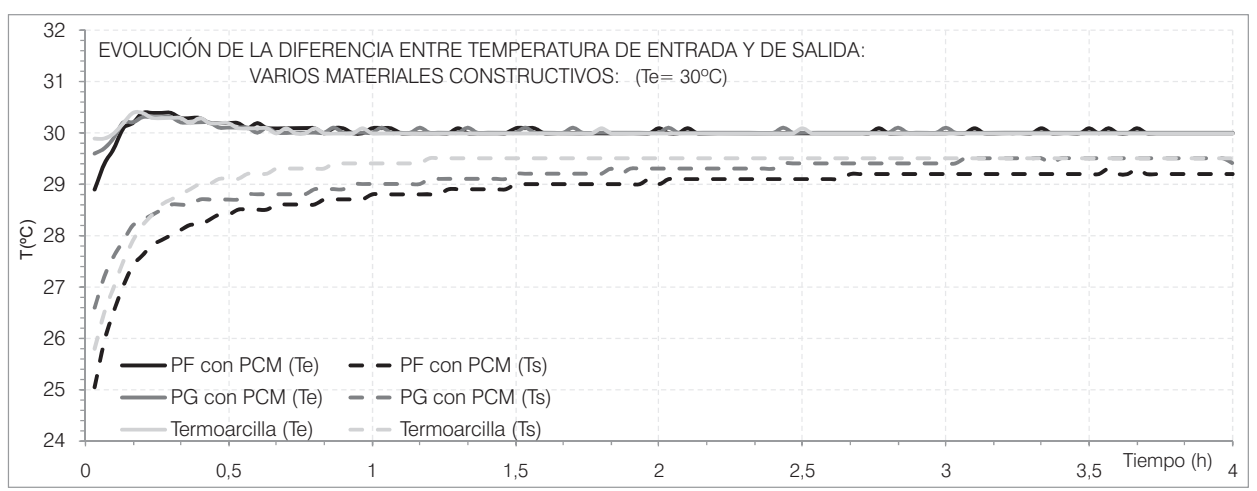


Gráfica 6. Comparación de la evolución de la diferencia entre Te y Ts, para varios materiales constructivos $\left(\mathrm{Te}, 30^{\circ} \mathrm{C}\right)$. Posición exenta

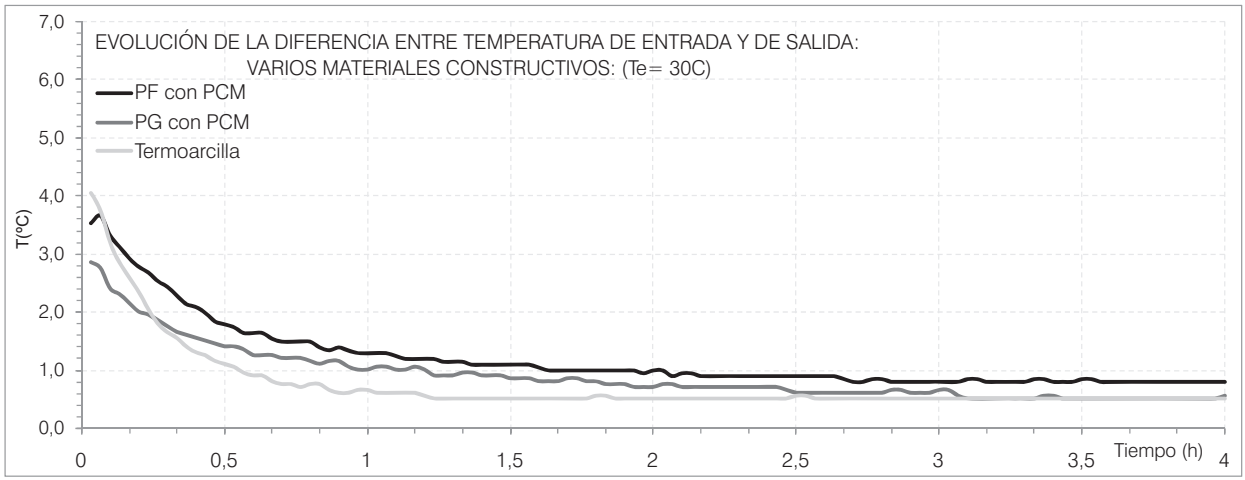

Gráfica 7. Comparación de la energía acumulada por intervalos, para varios materiales constructivos $\left(\mathrm{Te}, 30^{\circ} \mathrm{C}\right)$. Posición exenta

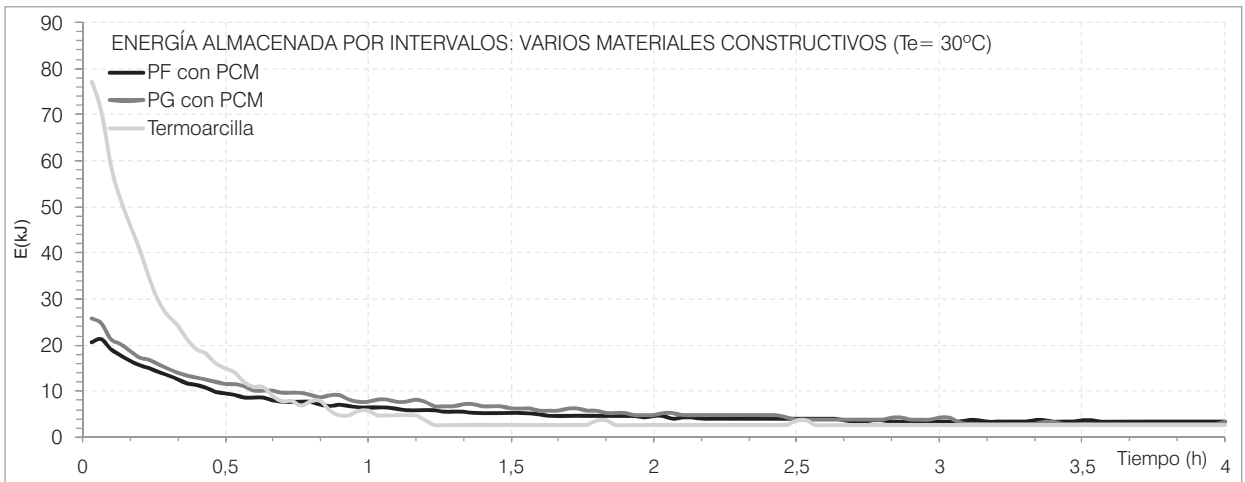

Gráfica 8. Comparación de la energía acumulada por $\mathbf{m}^{2}$ de superficie de materiales constructivos $\left(\mathrm{Te}, 30^{\circ} \mathrm{C}\right)$. Posición exenta

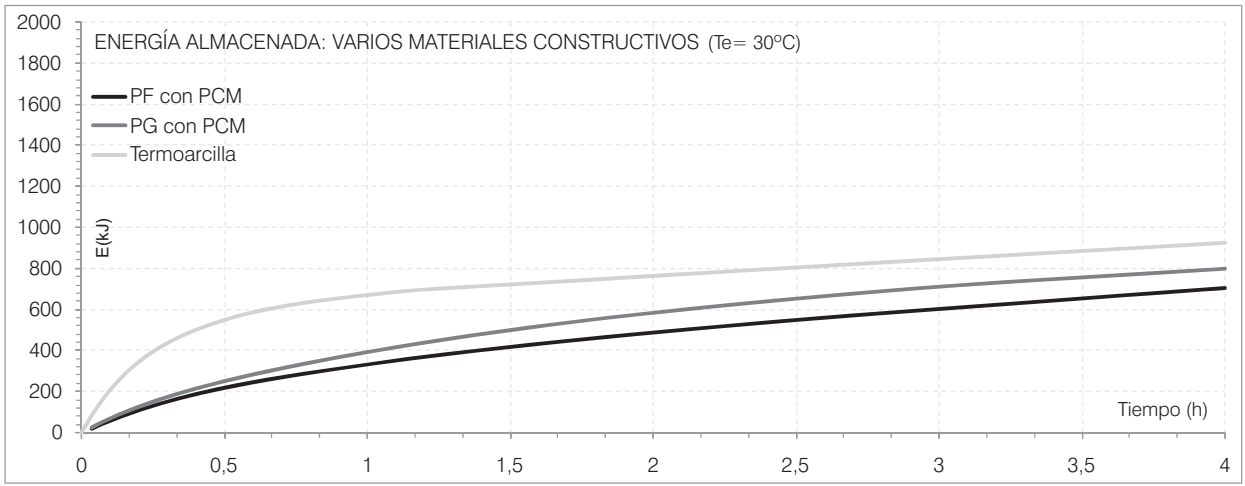

Tabla 2

Comparación de energía acumulada por $\mathrm{m}^{2}$ de superficie de materiales constructivos $\left(\mathrm{Te}, 30^{\circ} \mathrm{C}\right)$. Posición exenta

\begin{tabular}{ccc}
\hline \multicolumn{3}{|c|}{ Energía almacenada (kJ) } \\
\hline Material & \multicolumn{2}{c|}{ Tiempo } \\
\hline & $2 \mathrm{~h}$ & $4 \mathrm{~h}$ \\
$\mathrm{PF}$ & 489,04 & 705,06 \\
$\mathrm{PG}$ & 541,03 & 740,46 \\
$\mathrm{~T}$ & 644,87 & 929,46 \\
\hline
\end{tabular}

\section{Tabla 3}

Comparación de energía almacenada por $\mathrm{m}^{2}$ de de superficie de materiales constructivos $\left(\mathrm{Te}, 35^{\circ} \mathrm{C}\right)$. Posición trasdosada

\begin{tabular}{|c|c|c|}
\hline \multicolumn{3}{|c|}{ Energía almacenada $(\mathrm{kJ})$} \\
\hline \multirow{2}{*}{ Material } & \multicolumn{2}{|c|}{ Tiempo } \\
\hline & $2 \mathrm{~h}$ & $4 \mathrm{~h}$ \\
\hline PF & 407,54 & 587,55 \\
\hline PG & 727,81 & 1050,89 \\
\hline CY & 157,04 & 229,05 \\
\hline LH & 635,01 & 878,69 \\
\hline
\end{tabular}


rial de cambio de fase. Gran parte de esta energía se ha acumulado al inicio del ensayo cuando la diferencia entre temperaturas era superior.

Elevando la temperatura de trabajo a $35^{\circ} \mathrm{C}$, se obtienen los siguientes resultados:

Para el sistema trasdosado los valores obtenidos se representan en las Gráficas 9, 10, 11 y 12.

Esta temperatura $-35{ }^{\circ} \mathrm{C}-$ representa unas condiciones de contorno del interior de un edificio con grandes ganancias térmicas -radiación solar, equipos, ocupación, iluminación,...- y mejora la eficacia de las placas que contienen material de cambio de fase.

A esta temperatura las placas gruesas de yeso con PCM, almacenan un 13,8\% más que la fábrica de ladrillo hueco, un $22 \%$ más de energía que las placas finas $(1,5 \mathrm{~cm}$ de espesor), un $71,9 \%$ más que las placas de yeso laminado.

En efecto, tanto la fábrica de ladrillo como el yeso laminado, confían su capacidad térmica a la variación de temperatura del propio material, y a medida que esta tiende a 0 -cuando se estabiliza el sistema-, pierde efectividad el conjunto.
Para la posición exenta oblicua (con Te = $35{ }^{\circ} \mathrm{C}$ ), los valores se representan en las Gráficas 13, 14, 15 y 16.

En la Tabla 4 se reproducen los valores obtenidos en la Gráfica 16, a las 2 y 4 h. de inicio del ensayo.

Con estas condiciones de trabajo, las placas de $1,5 \mathrm{~cm}$ de espesor, almacenan un 17,3\% menos energía que las placas gruesas, mientras que la hoja de termoarcilla los supera en un $16 \%$.

\section{CONCLUSIONES}

\subsection{Comparación entre distintos materiales}

- Es posible determinar la velocidad de fusión o cambio de fase del material ya que depende del flujo de energía aportado, es decir, de la temperatura de la sala donde se encuentra colocado así como de la velocidad del aire que circula, especialmente el próximo al material (paredes).

- Conociendo las condiciones de contorno, como velocidad del aire en el interior de la sala, régimen de temperaturas de ésta, se puede calcular la cantidad de material microencapsulado necesario para que mediante el

Gráfica 9. Comparación de la evolución de la diferencia entre Te y Ts, para $1 \mathrm{~m}^{2} \mathrm{de}$ superficie de materiales constructivos $\left(\mathrm{Te}, 35^{\circ} \mathrm{C}\right.$ ). Posición adosada

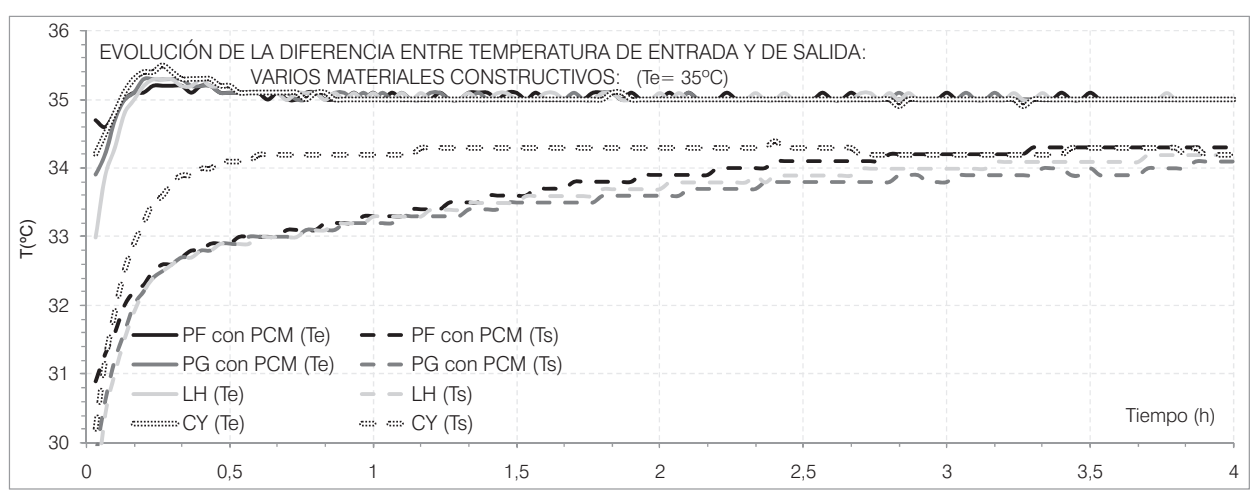

Gráfica 10. Comparación de la $\mathrm{T}$ interior de la placa para diferentes Te

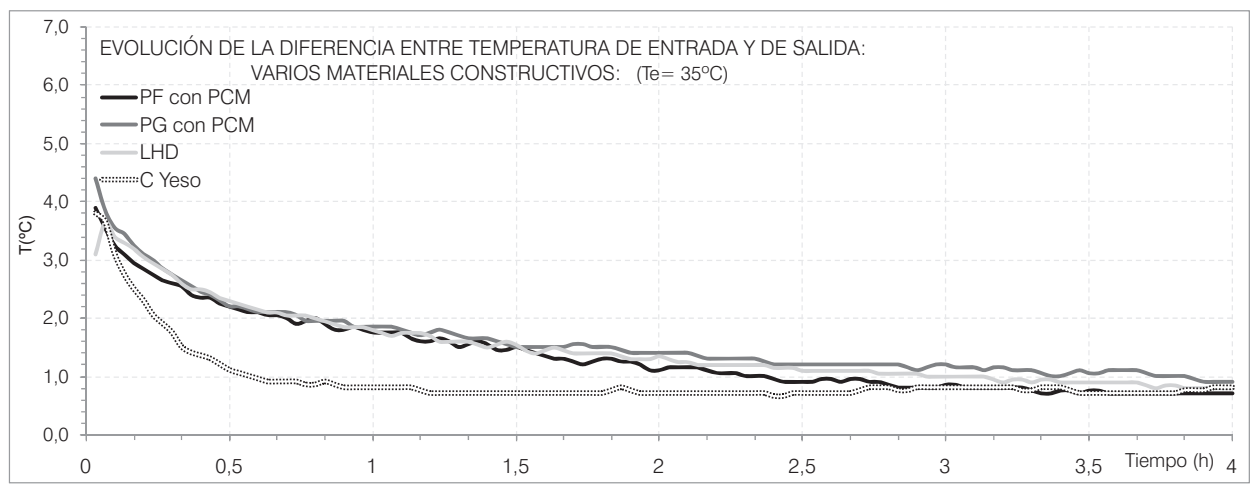


Gráfica 11. Comparación de la energía almacenada por intervalos para varios materiales constructivos ( $\mathrm{Te}, 35^{\circ} \mathrm{C}$ ). Posición adosada

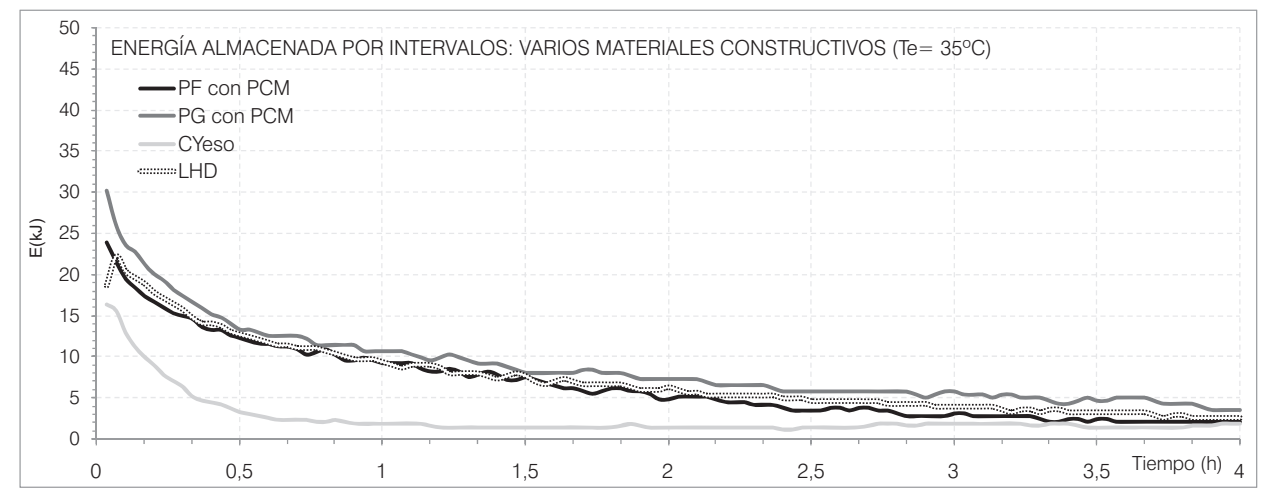

Gráfica 12. Comparación de la energía almacenada por diferentes materiales constructivos (Te, $\left.35^{\circ} \mathrm{C}\right)$. Posición adosada

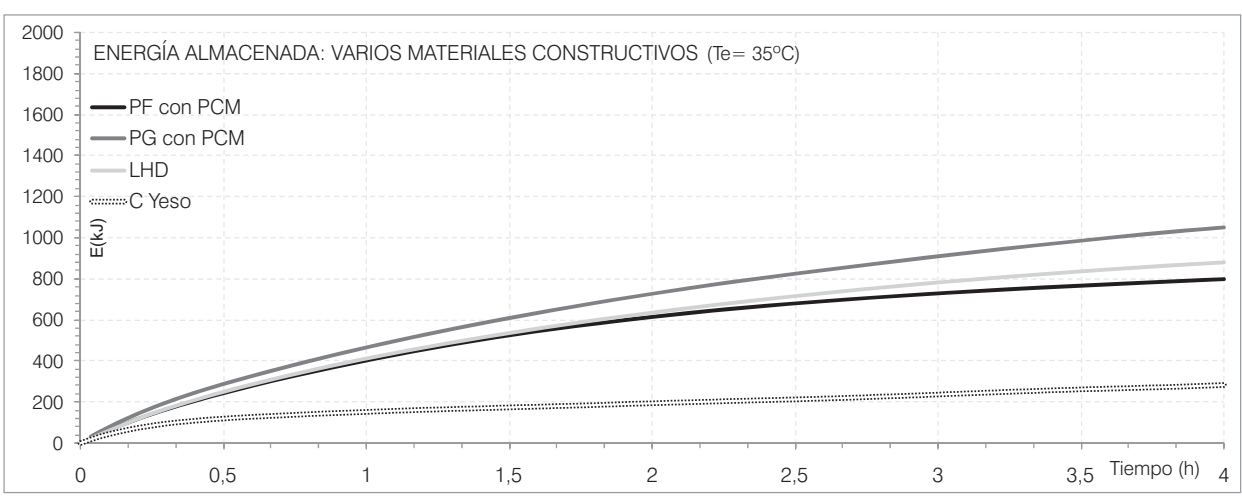

Gráfica 13. Evolución de Ts para $1 \mathrm{~m}^{2}$ de superficie de diferentes materiales constructivos $(\mathrm{PF}$, placas finas, $\mathrm{e}=1,5 \mathrm{~cm}, \mathrm{PG}$, placas gruesas,. $\mathrm{e}=2,5 \mathrm{~cm}$, ambos de escayola con Micronal al 44,5\%; Termoarcilla) (Te, $\left.35^{\circ} \mathrm{C}\right)$. Posición exenta

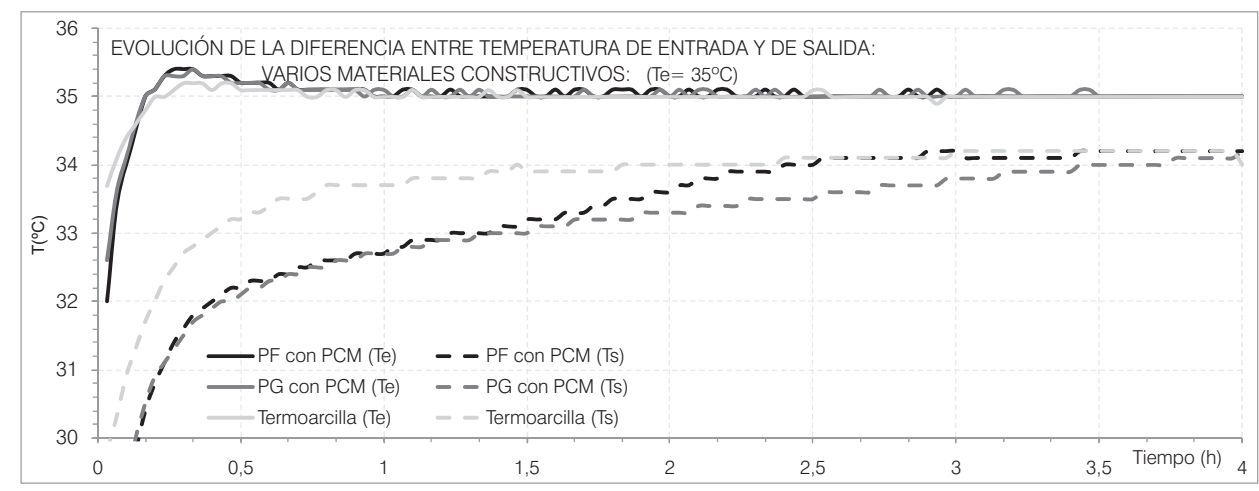

almacenamiento de calor latente -combinado con estrategias de calefacción y refrigeración pasivas-, contribuya a la minimización del consumo energético en edificación.

- Comparando la energía almacenada por unidad de superficie de cada elemento constructivo se obtiene la Tabla 5, pág. siguiente.

- A $30{ }^{\circ} \mathrm{C}$, una placa de yeso con PCM incorporado en un $44,5 \%$, de $1 \mathrm{~m}^{2}$ y $1,5 \mathrm{~cm}$
Tabla 4

Comparación de energía almacenada por $\mathrm{m}^{2}$ de superficie de materiales constructivos (Te, $\left.35^{\circ} \mathrm{C}\right)$. Posición exenta

\begin{tabular}{|c|c|c|}
\hline \multicolumn{3}{|c|}{ Energía almacenada (kJ) } \\
\hline \multirow{2}{*}{ Material } & \multicolumn{2}{|c|}{ Tiempo } \\
\hline & $2 \mathrm{~h}$ & $4 \mathrm{~h}$ \\
\hline PF con PCM & 592,34 & 740,73 \\
\hline PG con PCM & 1019,86 & 1402,77 \\
\hline Termoarcilla & 1302,15 & 1627,30 \\
\hline
\end{tabular}


Tabla 5

Comparación de energía almacenada por $\mathrm{m}^{2}$ de superficie de material constructivo

\begin{tabular}{|c|c|c|c|}
\hline \multirow{2}{*}{ MATERIAL } & \multicolumn{3}{|c|}{ Energía almacenada (kJ) } \\
\cline { 2 - 4 } & Te & $30{ }^{\circ} \mathrm{C}$ & $35{ }^{\circ} \mathrm{C}$ \\
\hline placas finas & $\mathrm{T}$ & $\mathbf{5 8 7 , 5 5}$ & $\mathbf{7 0 5 , 0 6}$ \\
\hline \multirow{2}{*}{ placas gruesas } & EO & $\mathbf{7 0 5 , 0 6}$ & $\mathbf{7 4 0 , 7 3}$ \\
\hline \multirow{2}{*}{ Yeso laminado } & $\mathrm{T}$ & $\mathbf{6 1 7 , 0 5}$ & $\mathbf{1 0 5 0 , 9}$ \\
\hline \multirow{2}{*}{ EO pie ladrillo } & $\mathrm{T}$ & $\mathbf{7 4 0 , 4 6}$ & $\mathbf{1 4 0 2 , 8}$ \\
\hline & EO & - & 229,05 \\
\hline termoarcilla & $\mathrm{T}$ & 641,69 & $\mathbf{8 7 8 , 6 9}$ \\
\hline & EO & - & - \\
\hline
\end{tabular}

fábrica de $14 \mathrm{~cm}$ de espesor de termoarciIla, a $35^{\circ} \mathrm{C}$.

- Considerando que $1 \mathrm{~m}^{2}$ de placa de yeso $(\mathrm{e}=1,5 \mathrm{~cm})$ pesa $10,68 \mathrm{~kg}$, y que la misma superficie, fabricada con termoarcilla $(\mathrm{e}=14 \mathrm{~cm})$, pesa $140 \mathrm{~kg}$, se ha elaborado la Tabla 6 para tener una referencia de la energía almacenada por unidad de masa.

- El retardo térmico que produce el material, y que está en relación con la velocidad de cambio de fase, sirve para compensar las diferencias de temperatura entre el día y la noche que se producen en los climas continentales como el de Madrid.

- De ello se concluye que para las mismas condiciones de ensayo almacena 5 veces más energía por unidad de masa que la

Gráfica 14. Comparación de la evolución de la diferencia entre Te y Ts, para varios materiales constructivos $\left(\mathrm{Te}, 35^{\circ} \mathrm{C}\right)$. Posición exenta

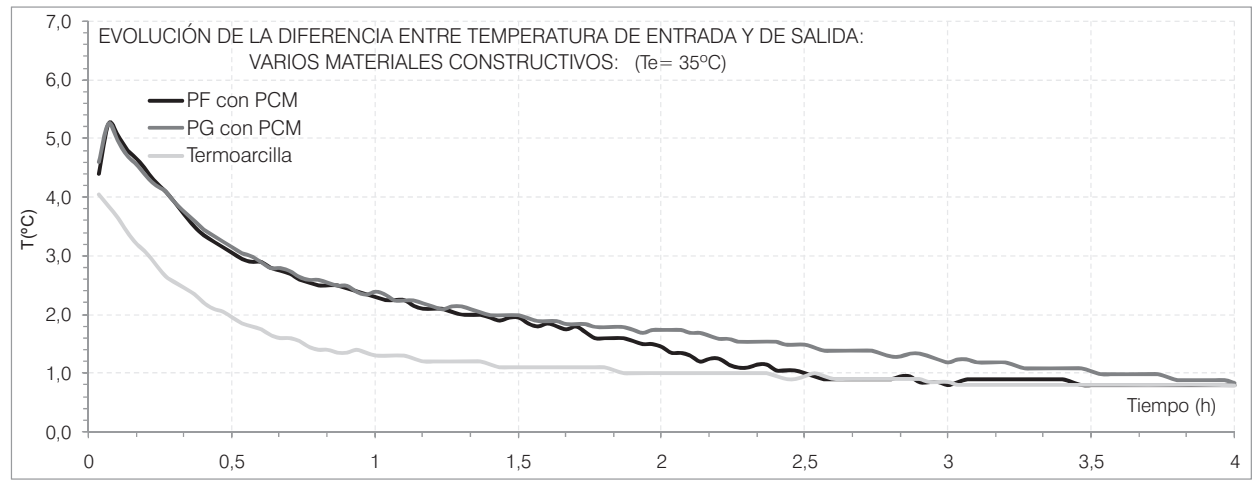

Gráfica 15. Comparación de la energía almacenada en cada intervalo, por $\mathbf{m}^{2}$ de superficie de material constructivo ( $\left.\mathrm{Te}, 35^{\circ} \mathrm{C}\right)$. Posición exenta

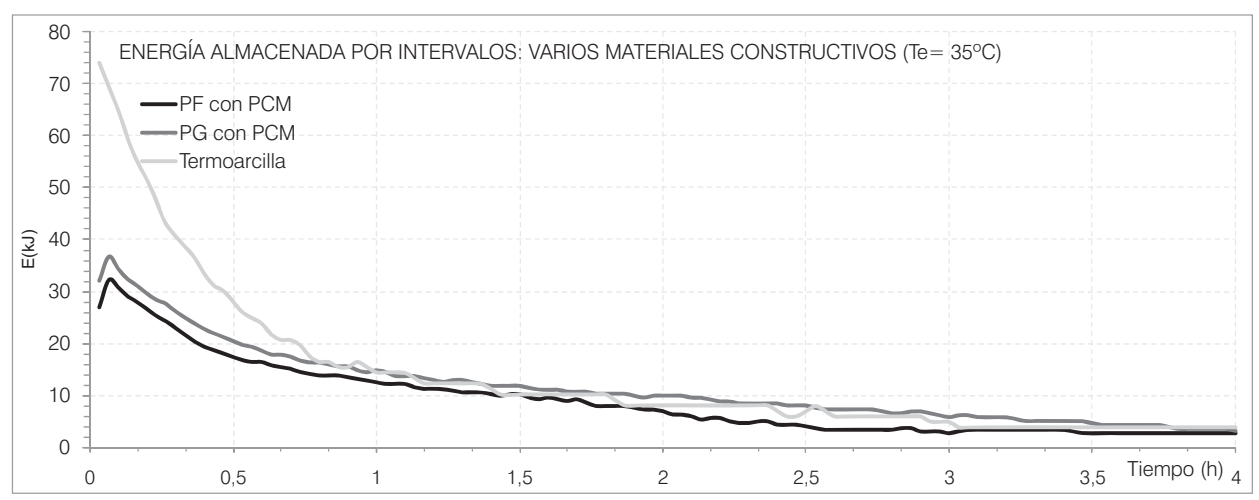

de espesor, almacena prácticamente la misma energía que una fábrica de $1 / 2$ pie de ladrillo hueco, y 5 veces la energía almacenada por una placa de yeso convencional del mismo espesor.

- Una placa de 2,5 cm de yeso con PCM, almacena casi la misma energía que una fábrica de termoarcilla; más de 9 veces lo que una fábrica de ladrillo y casi tres veces la energía por unidad de masa de una placa de yeso convencional. Así el compuesto y elemento constructivo queda caracterizado térmicamente. $Y$ la viabilidad y oportunidad para su fabricación y comercialización queda demostrada. 
Gráfica 16. Comparación de la energía almacenada por $\mathrm{m}^{2}$ de superficie de material constructivo $\left(\mathrm{Te}, 35^{\circ} \mathrm{C}\right)$. Posición exenta

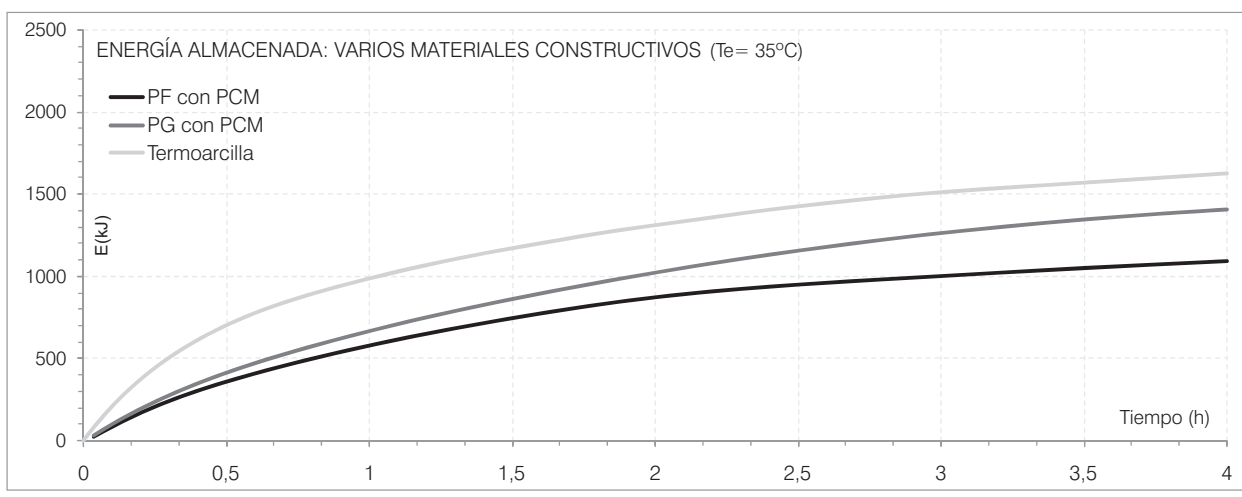

Tabla 6

Comparación de energía almacenada por unidad de masa de material constructivo

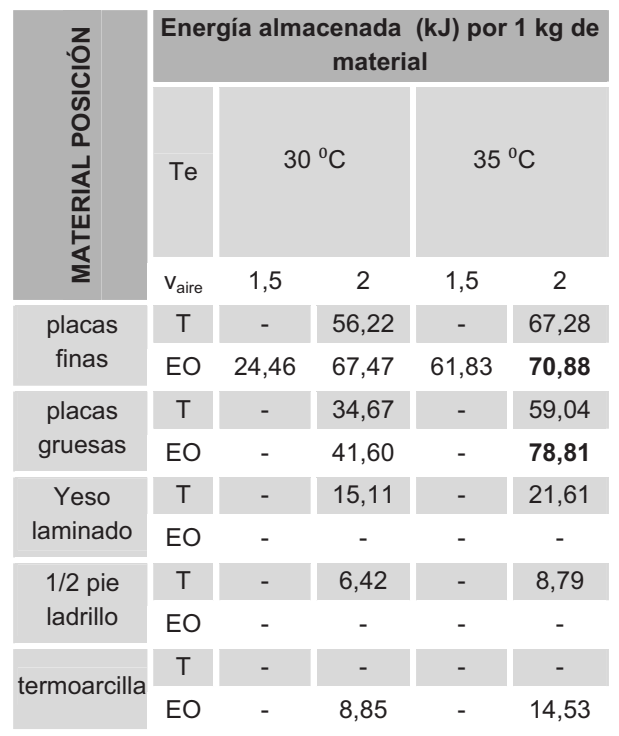

\section{BIBLIOGRAFÍA}

(1) Abhat, A. (1983). "Low temperature latent heat Thermal energy storage-Heat storage materials". Solar Energy 30 (4).

(2) Athientis A. K., L. C., Hawes D., Banu D., Feldman D. (1997). "Investigation of the Thermal performance of a passive solar test-room with wall latent heat storage". Building and Environment. 32: 405-410.

(3) BASF, www.basf.com.

(4) Bader, M. (2002) "Microencapsulated Paraffin in Polyethylene for Thermal Energy Storage". Department of Chemical \& Material Engineering, School of Engineering. the University of Auckland. New Zealand.

(5) Darkwa, K. and J. S. Kim (2004). "Heat transfer in composite laminated phase-change drywall". Proceedings of the institution of Mechanical Engineers, Part A: Journal of Power and Energy 218 (2): 83-87.

(6) Feldman D., B. D., Hawes D., Ghanbari E.(1991). "Obtaining an energy storing building material by direct incorporation of an organic phase change material in gypsum wallboard". Solar Energy Materials. 22 231-242.

(7) Hasan, A. and A. A. Sayigh (1994). "Some fatty acids as phase-change Thermal energy storage materials". Renewable energy 4 (1): 69-76.

(8) Hauer, A. (2002). "Innovative Thermal Energy Storage Systems for Residential Use", Bavarian Center for Applied Energy Research, ZAE Bayern: 8.

(9) Hawlader, M. N. A., M. S. Uddin, et al. (2003). "Microencapsulated PCM Thermal-energy storage system". Applied Energy 74 (1): 195-202. 
(10) Hunger M, Entrop AG, Mandilaras I, Brouwers HJH (2009) "The behavior of self-compacting concrete containing micro-encapsulated Phase Change Materials" Cement \& Concrete Composites 31.

(11) Koschenz M., Lehmann B. "Development of a thermally activated ceiling panel with PCM for application in lightweight and retrofitted buildings" Energy and Buildings 36 (2004) 567-578.

(12) Neeper, D. A. (2000). "Thermal dynamics of wallboard with latent heat storage". Solar Energy 68 (5): 393-403.

(13) Oliver, A. (2009). "Incorporación de Materiales de Cambio de Fase en Placas de Yeso Reforzadas con fibras de polipropileno. Aplicación a Sistemas de Calefacción y Refrigeración Pasivos para Almacenamiento de Calor Latente en Edificios". Tesis Doctoral.

(14) Salyer, I. O. (1999). "Phase Change Materials incorporated throughout the Structure of Polymer Fibers". US 5, 885, 475.

(15) Stetiu, C. and H. E. Feustel (1998). "Phase-Change Wallboard and Mechanical Night Ventilation in Commercial Buildings". Lawrence Berkeley National Laboratory.

(16) X Wang, YP Zhang, W Xiao, RL Zeng, QL Zhang (2009) "Review on thermal performance of phase change energy storage building envelope". Chinese Science Bulletin | March 2009 | vol. 54 | no. 6 | 920-928.

(17) Zalba, B. (2002). "Almacenamiento térmico de energía mediante cambio de fase". Procedimiento experimental. Departamento de ingeniería Mecánica. Zaragoza Universidad de Zaragoza.

(18) Y. Zhang et al. "Application of latent heat thermal energy storage in buildings: State-of-the-art and Outlook" (2008) / Building and Environment 42 (2007) 2197-2209. 